\title{
SOME HOMOTOPY OF STUNTED COMPLEX PROJECTIVE SPACE
}

\author{
BY ROBERT E. MOSHER
}

Communicated by F. P. Peterson, August 15, 1967

1. Introduction. The 2-components of the stable homotopy groups $\pi_{2 n+i}^{s}\left(C P / C P^{n-1}\right)$ of stunted complex projective space are here tabulated, up to group extension, for $8 \leqq i \leqq 13$. For earlier work, including computation of these groups for $i \leqq 7$, see [8], [2], [7], [3], [4], and [5] as corrected by [6]. See [1] for odd components.

A result of Toda [8] relates these stable groups to the metastable homotopy groups of unitary groups as follows: Let $0 \leqq t<n$. Then $\pi_{2 n+2 t+1}^{s}\left(C P / C P^{n-1}\right)=\pi_{2 n+2 t+1} U(n)$, while there exists a commutative diagram with an exact row

$$
\begin{aligned}
0 \longrightarrow Z & \longrightarrow \pi_{2 n+2 t}^{s}\left(C P / C P^{n-1}\right) \rightarrow \pi_{2 n+2 t} U(n) \rightarrow 0 \\
(n+t) ! & h \mid \\
Z & =H_{2 n+2 t}\left(C P / C P^{n-1}\right)
\end{aligned}
$$

in which $h$ is the Hurewicz homomorphism.

In view of Toda's formula the value of $h$ is needed to deduce $\pi_{2 n+2 t}^{-} U(n)$. We include this data as (2.3) and give in (2.5) the order of the image of each element of the 2-component of $\pi_{2 n+i}^{s} S^{2 n}$ in $\pi_{2 n+i}^{s}\left(C P / C P^{n-1}\right)$.

Our basic method is the stable homotopy exact couple resulting from the standard cell filtration of $C P / C P^{n-1}$. By naturality, differentials in the resulting spectral sequence for $C P / C P^{n-1}$ may be computed in the analogous spectral sequence for $C P$. The study in [6] of this sequence for $C P$ is the basis of the calculation here; a more detailed description of the calculation will appear elsewhere.

\section{Results on homotopy groups.}

THEOREM 2.1. The 2-component of the torsion of the stable homotopy group $\pi_{2 n+i}^{s}\left(C P / C P^{n-1}\right), 8 \leqq i \leqq 13$, is given by Table 2.2 .

In (2.2) $n Z_{2}$ denotes the direct sum of $n$ copies of $Z_{2}$, while $A$ ? $B$ denotes a group satisfying an exact sequence $0 \rightarrow A \rightarrow A$ ? $B \rightarrow B \rightarrow 0$. Note that $Z_{2}+Z_{2}$ ? $2 Z_{2}$ denotes $Z_{2}+A$, where $A=Z_{2}$ ? $2 Z_{2}$, rather 


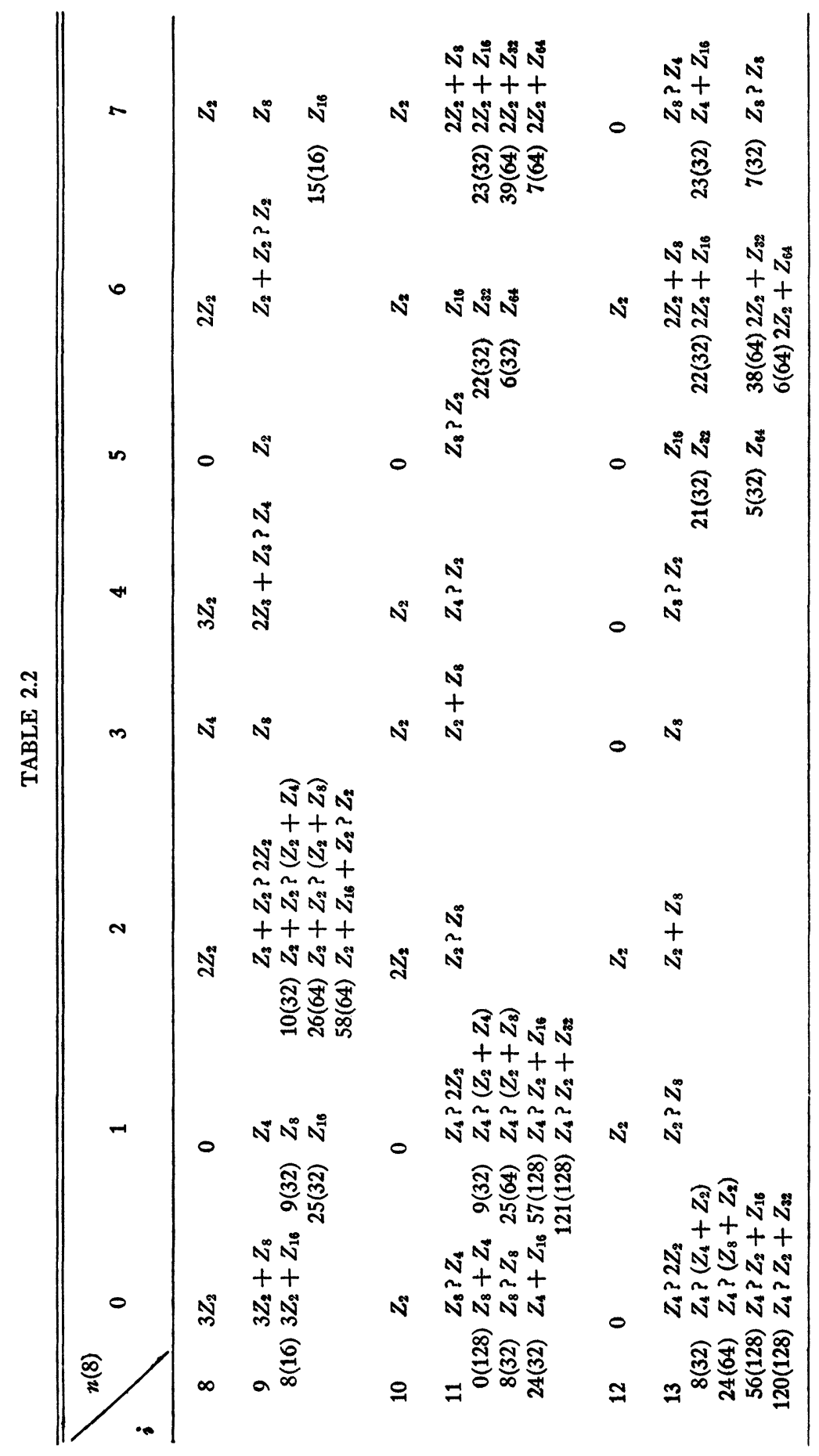


than $2 Z_{2}$ ? $2 Z_{2}$. The multiple entry for $i=9$ and $n \equiv 1(8)$ should be read as $Z_{4}$ except for $n \equiv 9(32)$ and $n \equiv 25(32)$.

Modulo torsion, $\pi_{2 n+i}^{s}\left(C P / C P^{n-1}\right)$ vanishes for $i$ odd, but is infinite cyclic for $i$ even.

THEOREM 2.3. Let $x_{n+k}$ generate $H_{2 n+2 k}\left(C P / C P^{n-1}\right)$. Let $h_{n+k, k} x_{n+k}$ generate the image of the Hurewicz homomorphism $h: \pi_{2 n+2 k}^{s}\left(C P / C P^{n-1}\right)$ $\rightarrow H_{2 n+2 k}\left(C P / C P^{n-1}\right)$. Then, up to multiplication by an odd integer, for $1 \leqq k \leqq 6 h_{n+k, k}$ is given by Table 2.4 , while $h_{n+7,7}=h_{n+7,6}$.

TABLE 2.4 .

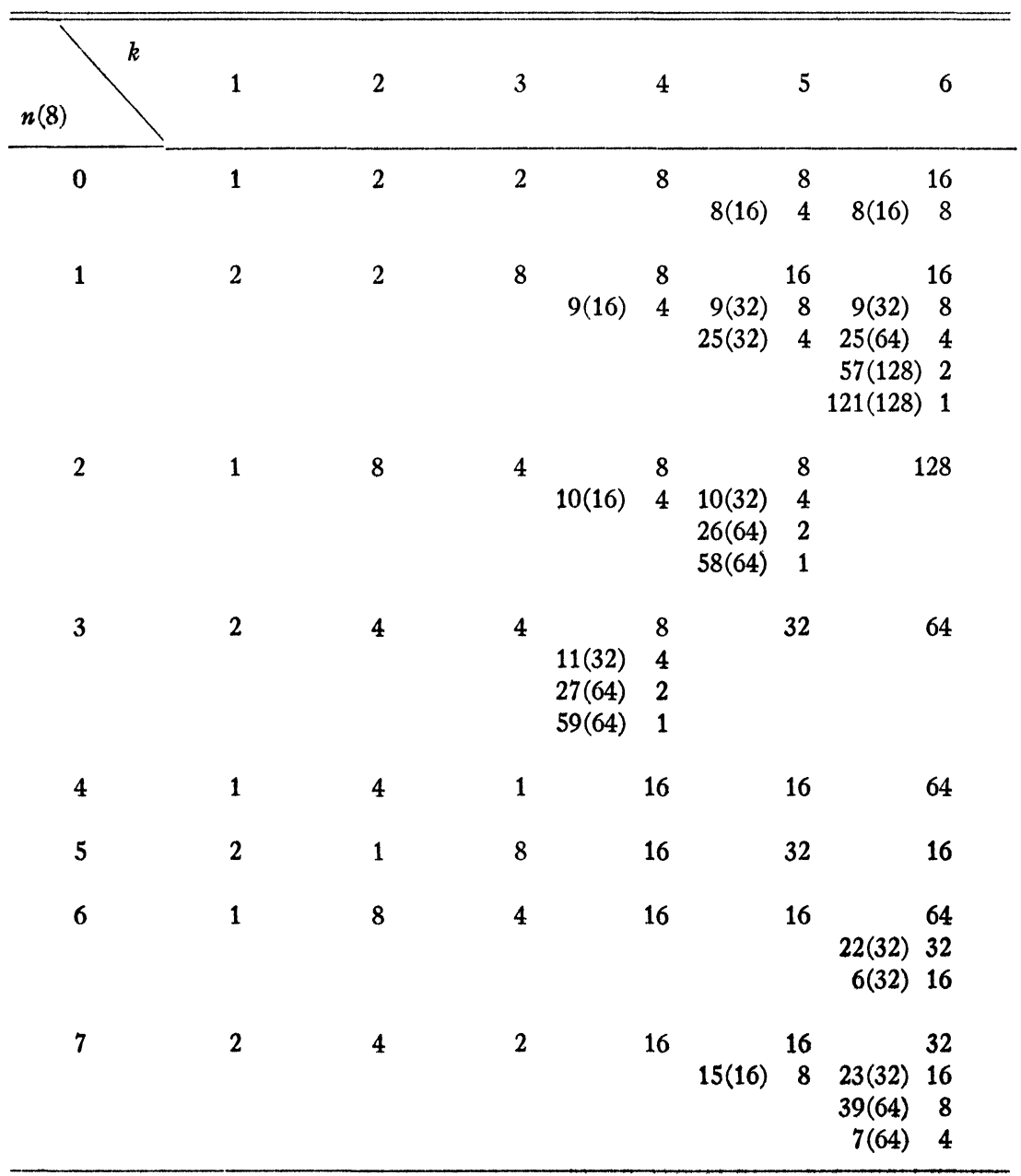


THEOREM 2.5. Let $\beta$ be in the 2-component of $G_{i}, 0<i \leqq 13$. Let $j: S^{2 n} \rightarrow C P / C P^{n-1}$ be the inclusion. Then the order of $j * \beta \in \pi_{2 n+i}^{s}$ $\cdot\left(C P / C P^{n-1}\right)$ is given by Table 2.6.

Nomenclature for elements of $G$, the stable homotopy of spheres, is as in [9].

TABLE 2.6.

\begin{tabular}{|c|c|c|c|c|c|c|c|c|c|c|c|c|c|c|}
\hline$n(8)$ & $\eta$ & $\eta^{2}$ & $\nu$ & $\nu^{2}$ & $\sigma$ & $\epsilon$ & $\vec{\nu}$ & $\eta \sigma$ & $\eta \epsilon$ & $\eta \bar{\nu}$ & $\eta^{2} \sigma$ & $\mu$ & $\eta \mu$ & $\zeta$ \\
\hline 0 & 2 & 2 & 8 & 2 & 16 & 2 & 2 & 2 & 2 & 2 & 2 & 2 & $\begin{array}{c}2 \\
24(32)\end{array}$ & $\begin{array}{l}8 \\
4\end{array}$ \\
\hline 1 & 0 & 0 & 2 & $\begin{array}{c}2 \\
9(16)\end{array}$ & $\begin{array}{r}8 \\
16\end{array}$ & 0 & 0 & 0 & 0 & 0 & $\begin{array}{c}0 \\
25(32)\end{array}$ & $\begin{array}{l}0 \\
212\end{array}$ & $\begin{array}{c}0 \\
21(128)\end{array}$ & $\begin{array}{l}2 \\
4\end{array}$ \\
\hline 2 & 2 & 2 & 2 & $\begin{array}{c}0 \\
10(16)\end{array}$ & $\begin{array}{r}8 \\
16\end{array}$ & 2 & 2 & 2 & 2 & 0 & 2 & 2 & 2 & 2 \\
\hline 3 & 0 & 0 & 0 & $\begin{array}{c}0 \\
11(32) \\
27(64) \\
59(64)\end{array}$ & $\begin{array}{r}2 \\
4 \\
8 \\
16\end{array}$ & 2 & 2 & 0 & 0 & 0 & 0 & 0 & 0 & 0 \\
\hline 4 & 2 & 2 & 4 & 2 & 8 & 2 & 2 & 2 & 2 & 2 & 2 & 2 & 2 & 4 \\
\hline 5 & 0 & 0 & 4 & 2 & 4 & 0 & 0 & 0 & 0 & 0 & 0 & 0 & 0 & 4 \\
\hline 6 & 2 & 2 & 2 & 0 & 2 & 2 & 2 & 2 & 2 & 0 & 2 & 2 & $\begin{array}{c}2 \\
22(32) \\
6(32)\end{array}$ & $\begin{array}{l}0 \\
4 \\
8\end{array}$ \\
\hline 7 & 0 & 0 & 0 & 0 & 2 & 0 & 0 & 0 & 0 & 0 & $\begin{array}{c}0 \\
15(16)\end{array}$ & $\begin{array}{l}0 \\
2\end{array}$ & $\begin{array}{c}0 \\
39(64) \\
7(64)\end{array}$ & $\begin{array}{l}0 \\
2 \\
4\end{array}$ \\
\hline
\end{tabular}

\section{REFERENCES}

1. H. Imanishi, Unstable homotopy groups of classical groups (odd primary components) (to appear).

2. M. A. Kervaire, Some nonstable homotopy groups of Lie groups, Illinois J. Math. 4 (1960), 161-169.

3. H. Matsunaga, The homotopy groups $\pi_{2 n+i}(U(n))$ for $i=3,4$, and 5, Mem. Fac. Sci, Kyushu Univ. Ser. A 15 (1961), 72-81.

4. - On the groups $\pi_{2 n+7}(U(n))$, odd primary components, Mem. Fac. Sci. Kyushu Univ. Ser. A 16 (1962), 68-74. 
5. - Applications of functional cohomology operations to the calculus of $\pi_{2 n+i}(U(n))$ for $i=6$ and 7, $n \geqq 4$, Mem. Fac. Sci. Kyushu Univ. Ser. A 17 (1963), 29-62.

6. R. E. Mosher, Some stable homotopy of complex projective space, Topology (to appear).

7. M. Rothenberg, The J functor and the nonstable homotopy groups of the unitary groups, Proc. Amer. Math. Soc. 15 (1964), 264-271.

8. H. Todo, $A$ topological proof of the theorems of Bott and Borel-Hirzebruch for homotopy groups of unitary groups, Mem. Coll. Sci. Kyoto 32 (1959), 109-119.

9. H. Toda, Composition methods in homotopy groups of spheres, Ann. of Math. Studies 49, Princeton Univ. Press, Princeton, N. J., 1962.

California State College at long Beach and

NORTHWESTERN UNIVERSITY

\section{SOME PROPERTIES OF DISTRIBUTIONS WHOSE PARTIAL DERIVATIVES ARE REPRESENTABLE BY INTEGRATION ${ }^{1}$}

BY HERBERT FEDERER

Communicated August 21, 1967

We denote $n$ dimensional Euclidean space by $R^{n}$ and let $H^{m}$ be $m$ dimensional Hausdorff measure.

It is well known that distributions of the type described in the title may alternately be characterized as corresponding to $H^{n}$ measurable real valued functions $f$ with the following property: There exists a sequence of infinitely differentiable real valued functions $f_{j}$ on $R^{n}$ such that

$$
\lim _{j \rightarrow \infty} \int_{K}\left|f_{j}-f\right| d H^{n}=0 \text { and } \liminf _{j \rightarrow \infty} \int_{K}\left\|D f_{j}\right\| d H^{n}<\infty
$$

for every compact subset $K$ of $R^{n}$. The class of such functions $f$ is now widely regarded as the proper generalization to $n>1$ of the class of those functions on $R$ which are $H^{1}$ equivalent to functions with finite total variation on every compact interval. However up to now there has been lacking an extension to $n>1$ of the basic classical results describing the continuity properties of functions with locally finite variation, namely that the set of points of discontinuity is countable and that one-sided limits exist everywhere. At first sight such an

1 This work was supported in part by a research grant from the National Science Foundation. 